\title{
Alignment of teeth using different aesthetic orthodontic techniques (conventional buccal technique, lingual technique and Inman Aligner): In vitro study \\ Received: $16 / 11 / 2014$ \\ Accepted: $16 / 3 / 2015$
}

Saad Sami Gasgoos*
Rawand Ahmed Mahmood *

\section{Abstract}

Background and objective: An expectation of beautiful smiles at the end of orthodontic treatment is a primary concern to each patient, but is also equally concerned with appearance while undergoing treatment. The development of appliances that combine both acceptable aesthetics for the patient and adequate technical performance for the clinician is the ultimate goal. The aim of this study was to compare three different aesthetic orthodontic techniques with different biomechanics in order to quantify the efficiency of these techniques in alignment of displaced upper right central incisor and investigating the effects of these variables on alignment considering its tipping and rotation.

Methods: The sample consisted of three groups with each group including 15 samples; (1) conventional buccal technique group, (2) lingual technique group and (3) Inman Aligner group, all on a typodont simulation system (dentsply). Preoperative and postoperative digital images were taken and analyzed using Autodesk AutoCAD software.

Results: The conventional buccal technique gave rise to the highest mean of tooth tipping and rotation followed by the lingual technique. The Inman Aligner produced the least level of tooth tipping and rotation.

Conclusion: The best method of alignment is the conventional buccal technique followed by the lingual technique. The least favourable one is the Inman Aligner.

Keywords: University of Mosul, College of Dentistry, Mosul.

\section{Introduction}

An expectation of beautiful smiles at the end of orthodontic treatment is a primary concern to each patient, but is also equally concerned with appearance while undergoing treatment. Many attempts have been made by manufacturers to meet this demand. ${ }^{1}$ In the mid 1980 s, the first brackets made of monocrystalline sapphire and polycrystalline ceramic materials came into the field of orthodontics. ${ }^{2,3}$ They were introduced as an esthetic appliance which, unlike plastic brackets, could withstand most orthodontic forces and resist staining. However, inability to form chemical bonds with resin adhesives, low fracture toughness and increased frictional resistance between metal archwires and ceramic brackets were remained as major disadvantages with ceramic brackets., ${ }^{2,4}$ Recently, a new ceramic bracket design having metal-lined arch wire slot was introduced. The advantage of having a stainless steel slot was to minimize the increased friction and the metal slot also helped strengthen the bracket in order to withstand routine orthodontic torque forces. ${ }^{5}$ As the number of adults seeking orthodontic treatment increased, demand for esthetic brackets was raised. Lingual orthodontics has become an alternative treatment technique for those patients who would like to have a beautiful smile but were concerned about unsightly labial brackets. This technique created interest and enthusiasm in the early 1980's. At that time, many orthodontists hastened to treat patients with the prototype lingual appliance, but soon became discouraged with lingual orthodontics because of a myriad of technical problems. ${ }^{6}$ Clinical protocols had not been fully elucidated in

* Department of P.O.P, college of dentistry, Mosul University, Mosul, Iraq. 
those early days, resulting in many clinicians feeling impelled to begin lingual orthodontic cases without being fully prepared. Orthodontists found that the new lingual technique required much more rigorous attention to detail, as well as a fundamentally different approach to treatment planning and biomechanics. ${ }^{6}$ A major objection to fixed orthodontic appliances always has been their visible placement on the facial surface of the teeth. This is one reason for using removable appliances, and is the major reason for the current popularity of clear aligners in treatment of adults. ${ }^{7}$ In developing the Inman Aligner, Inman Orthodontic Laboratory has created a patented design that takes advantage of the gentle, steady and consistent forces generated by nickel titanium (NiTi). The design relies on piston-like components driven by $\mathrm{NiTi}$ coil springs. The forces are varied by altering the coil size and/or compressing the coil to create a stronger force. Don Inman has also designed lingual and labial to function or move in parallel to the occlusal plane, eliminating the mousetrap like deseating forces and allowing true bodily movement of teeth. ${ }^{8}$ The aim of this study was to compare three different aesthetic orthodontic techniques with different biomechanics in order to quantify the efficiency of these techniques in alignment of displaced upper right central incisor and investigating the effects of these variables on alignment considering its tipping and rotation.

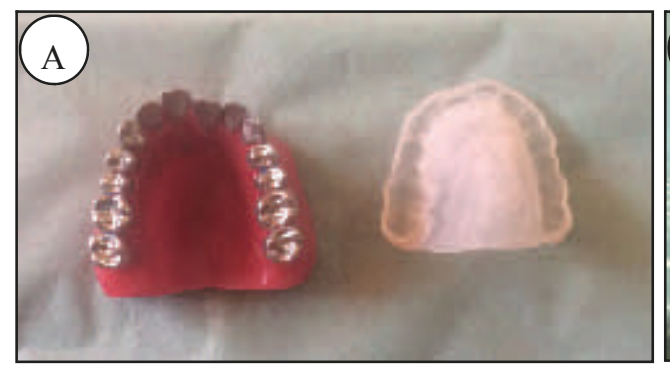

\section{Methods}

The samples of this study were divided into three groups with 15 samples per each group; group (1) conventional buccal technique, group (2) lingual technique and group (3) Inman Aligner. Typodont setup is done according to manufacturer instructions (Dentsply, Japan):

1. Remove the top and bottom metal plates and insert the wax form. Press the wax form firmly in place so that the wax is flush with articulator base. No further waxing of the base should be required. Replace the metal plates.

2. Note the palmer tooth number identification on the root, this will assist in properly identifying the teeth. Insert the teeth into their sockets in the wax form. Press the teeth firmly in to the wax.

3 . Once the teeth have been tried in the wax form, remove each tooth at a time for application of sticky wax.

4. Apply small amount of sticky wax around the roots of the teeth and this will prevent unwanted extrusion of the teeth.

In order to construct an Inman Aligner, an impression of the typodont teeth was obtained using silicon impression material; the impression was sent to a laboratory, where the customised Inman Aligner was fabricated according to the case.

For teeth rearrangement before each trial, an impression was taken with silicon impression material (heavy body and light body) and an acrylic guide plate was constructed. Another acrylic blocks were constructed for immobilisation of anchorage teeth (Figure 1).

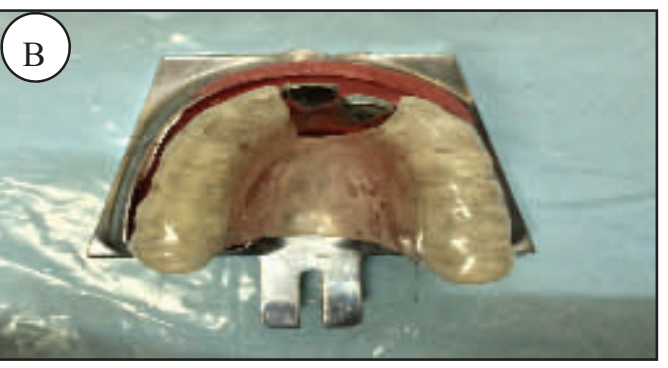

Figure 1: (A) Acrylic guide plate help in rearrangement of teeth after each movement or trial. (B) Sample of an acrylic block help in immobilisation of anchorage teeth after bracket placement. 
A special two stainless steel bars (rods) were constructed; the first was a bite plane extension bar (BPB) and the other was incisors extension bar (IB). BPB is an L-shape bar made from stainless steel rectangular wire of size $(0.018 \times 0.025$ inch). The vertical short arms are inserted in a hole made in the acrylic bite, then fixed with epoxy steel adhesive. These arms emerge upward for $20 \mathrm{~mm}$ distance. Then it bends to extend buccolingually (horizontally) making intersection angle of 90 degree with incisors extension bars both laterally and occlusally. IB are L-shape bars made from stainless steel rectangular wire of size $0.018 \times 0.025$. The long arm is bonded to the labial surface of incisors when using Inman Aligner or lingual technique and to the palatal surface of incisors in labial or conventional technique. It extends upward for $10 \mathrm{~mm}$ distance and then bends anteroposteriorly $5 \mathrm{~mm}$ above the incisal surface of the teeth and $5 \mathrm{~mm}$ below bite plane extension bar. These two bars are used as a guide for determining position, degree of tipping, degree of rotation and vertical height following alignment of the central incisors. This method is a modification of Huffman and Way procedure. ${ }^{9}$ A wooden table was constructed with two metallic bases, one to fix the digital camera (vertically for rotational measurements and horizontally for tipping measurements), and the other to fix the typodont in a way to allow a standard position for taking images before and after each movement (Figure 2). Pre and postoperative images for all samples were processed by Autodesk AutoCAD software. AutoCAD is a software application for computer-aided design (CAD) and drafting in both $2 \mathrm{D}$ and 3D. After each activation of a single appliance, the typodont was immersed in the water bath at controlled temperature of about $50 \pm 2^{\circ} \mathrm{C}$ for 5 minutes. ${ }^{10,11}$ In order to avoid the possible alteration of the characteristics of the wax, the wax was replaced after each trial. Rewax, lute, around the roots and crown of the teeth after each trial using the additional wax bar accessories (Dentsply, Japan). Typodont should not be left under the heat lamp for more than 5 minutes.

Measurements: Pre and post operative digital photographs of all techniques were analyzed using Autodesk AutoCAD software and all the measurements were calculated by subtracting the postoperative reading from the preoperative reading as shown in Figure (3, 4 and 5 ) according to the following equation. ${ }^{12,13}$

Variable reading $=$ preoperative reading postoperative reading

Measurements were made according to Huffman and Way method (1983) with some modifications that's simplified the method. A typodont is photographed with transverse projection from both right and left sides of typodont and with a vertical

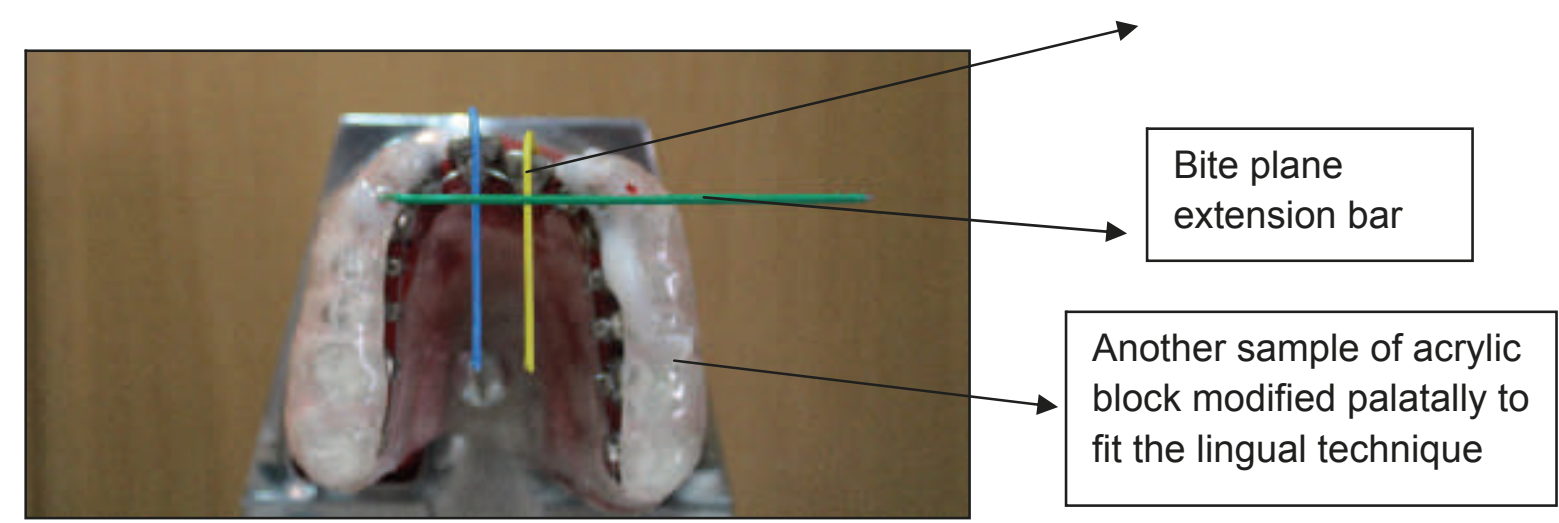

Figure 2: Incisors and bite plane extension bars. 
projection where the angle between IEB and PEB is exposed and then can be measured directly on the photograph. Statistical analysis of collected laboratory measurements was done using the statistical package for the social sciences (version 19). Descriptive statistics using the mean and standard deviation was applied. One way ANOVA test was performed for each group to assess the difference between them. A $P$ value of $\geq 0.05$ was considered statistically significant.
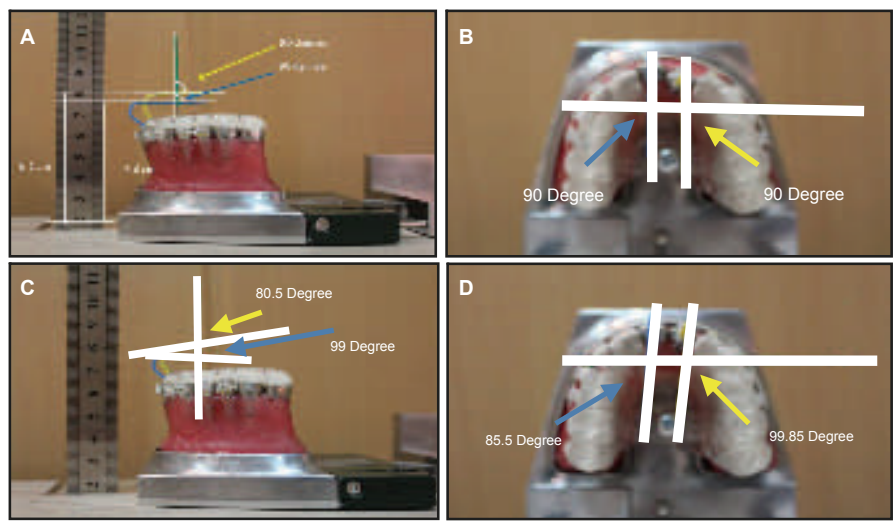

Figure 3: $(A, B)$ : Preoperative images of conventional buccal technique. (C,D): Postoperative images of conventional buccal technique showing tipping and rotation.
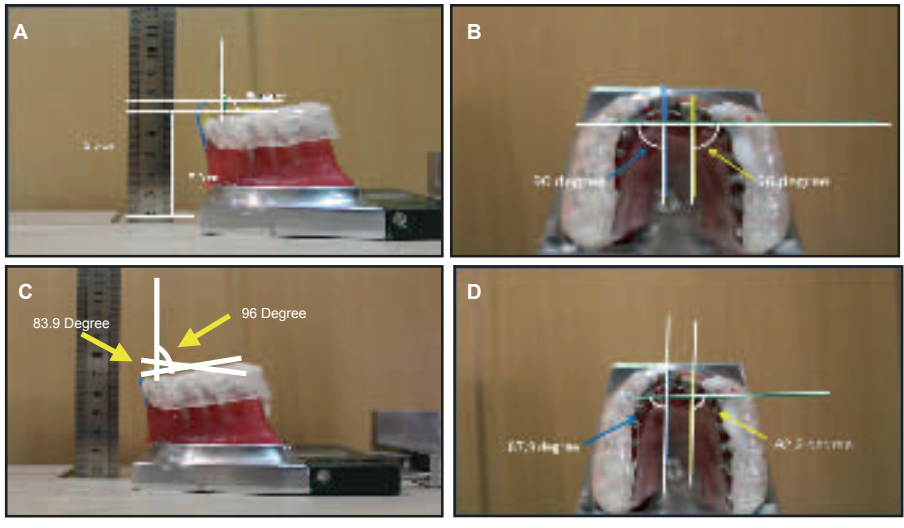

Figure 4: (A, B): Preoperative images of lingual technique. (C, D): Postoperative images of lingual technique showing tipping and rotation.
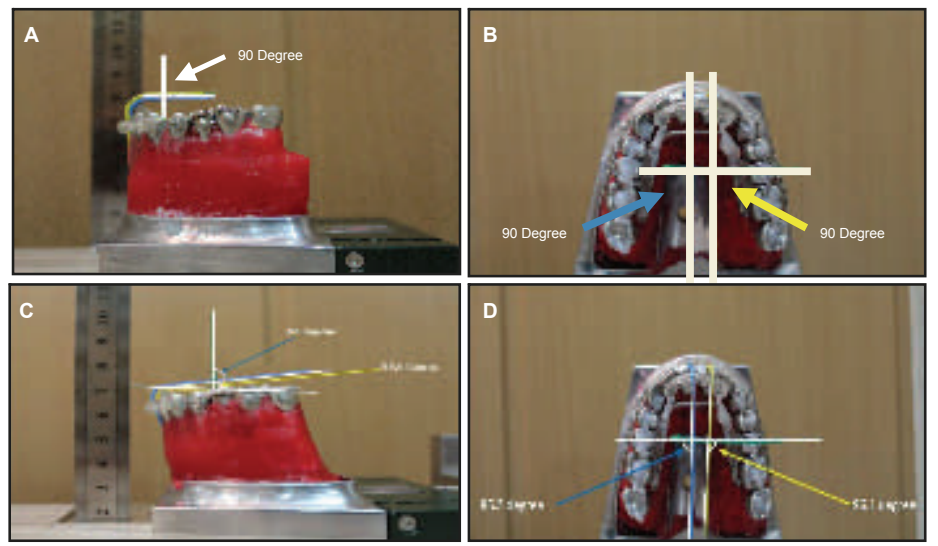

Figure 5: (A,B): Preoperative images of Inman Aligner. (C,D): Postoperative images of Inman Aligner showing tipping and rotation. 


\section{Results}

The mean and standard deviation for the degree of tipping, rotation and rate of space closure of the three groups are shown in Table 1.

Tipping: The Duncan Multiple Analysis Range Test showed that the conventional buccal technique had the highest amount of tipping with a significant difference from other groups, while Inman Aligner had the lowest level of tipping.

Rotation: The Duncan Multiple Analysis Range Test showed that conventional buccal technique had the highest amount of rotation with a significant difference from other groups, while Inman Aligner had the lowest level of rotation with a significant difference from other groups.

\section{Discussion}

Conventional buccal technique shows the highest mean of teeth tipping and rotation compared to lingual technique and Inman Aligner. Differences between the labial and lingual techniques have an important impact on the biomechanics of lingual orthodontics (Li O). These appliances have built in mechanical differences. ${ }^{14-16}$ First, the relationship between the point of force $(\mathrm{PF})$ and the centre of resistant (Cres) is different between labial orthodontics ( $\mathrm{LaO}$ ) and LiO because of the different position of the brackets (Figure 6).

Table 1: Descriptive analysis (mean and standard deviation) and $P$ value for tipping and rotation of upper right central incisor.

\begin{tabular}{llllll}
\hline & Groups & N & Mean* & SD & $P$ value \\
\hline Tipping & InRT & 15 & -7.0468 & 0.27112 & \\
& LiRT & 15 & -8.4716 & 0.33676 & $0.001<$ \\
& BuRT & 15 & -10.4027 & 0.33335 & \\
\multirow{5}{*}{ Rotation } & InRT & 15 & +3.1512 & 0.21967 & \\
& LiRT & 15 & +3.5050 & 0.24232 & $0.001<$ \\
& BuRT & 15 & +4.9000 & 0.27495 & \\
\hline
\end{tabular}

(In): Inman Aligner group.

(Bu): Conventional buccal technique group.

$(-)$ : Refer to inward movement of tooth.

$\left(^{*}\right)$ : Mean is measured in degree.
(Li): Lingual appliance group.

(RT): Right central incisor.

$(+)$ : Refer to anticlockwise rotation of tooth.

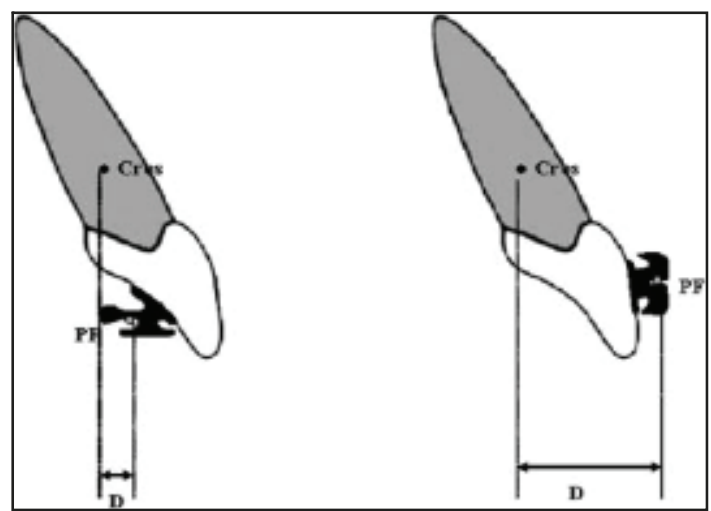

Figure 6: distance between center of resistant and point of force application in an incisor with labial and lingual bracket. ${ }^{14}$ 
Because the distance (D) between Cres and $\mathrm{PF}$ is smaller in $\mathrm{LiO}$ than in $\mathrm{LaO}$, the moments of forces in LiO are smaller (moment $=$ force $\times$ distance). As a consequence, torque is more difficult to control in LiO, and first-, second-, and third-order movements (in-out, extrusionintrusion, and torque) are also influenced. ${ }^{14}$ Second, the arch perimeter in the anterior region of the lingual tooth aspect is smaller than in the labial aspect. Consequently, the load deflection rate (L/D of the wire) in $\mathrm{LiO}$ is higher, which will make it more difficult to apply light optimal forces. Activation range and force constancy are reduced. Because increasing the wire length for reducing the $\mathrm{L} / \mathrm{D}$ rate is impossible in $\mathrm{LiO}$, the preferable options are to decrease the modulus of elasticity by using shape memory alloys and to reduce the cross section of the wire (Figure 7 ). ${ }^{17}$ Third, the lingual tooth aspect is more complex and versatile, and, therefore, every change in the bracket position on the lingual side may cause unpredictable and extensive change in the torque and vertical tooth height (Figure 8$).{ }^{18}$ The lingual brackets are

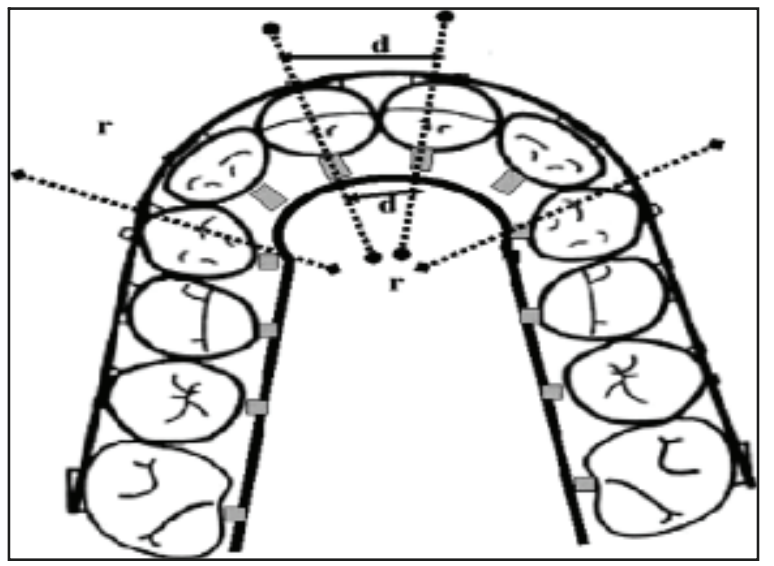

Figure 7: Arch perimeter and interbracket distance in a labial and lingual bracket system. Lateral shift of lingual brackets has a greater effect on the final tooth position than when the same shift is applied on labial brackets. ${ }^{14}$ bonded distally from the labial surface that has to be aligned. Torque can be greatly influenced by the lingual anatomy, especially in the maxillary incisors. A small variation in the incisogingival location of a bracket on a sloped lingual surface can significantly change the torque delivered to the tooth, whereas height variations on labial surfaces change the torque minimally. ${ }^{19-21}$ Fourth, the force moment within the bracket slot generated by the interaction between the archwire and the bracket slot is critical for the force exerted from the archwire to be transferred to the malpositioned tooth. ${ }^{22}$ The size of the archwire is important to generate the necessary moment. ${ }^{7,23}$ In the circumstances of tipping and rotation, the two-point contacts are located at the marginal edges of the bracket slot; the bracket width therefore is critical to induce adequate force moment ${ }^{23}$ (Note that the labial bracket is wider than lingual bracket). The correction of severely rotated and tipped teeth was found to be difficult using small-size brackets. ${ }^{24}$

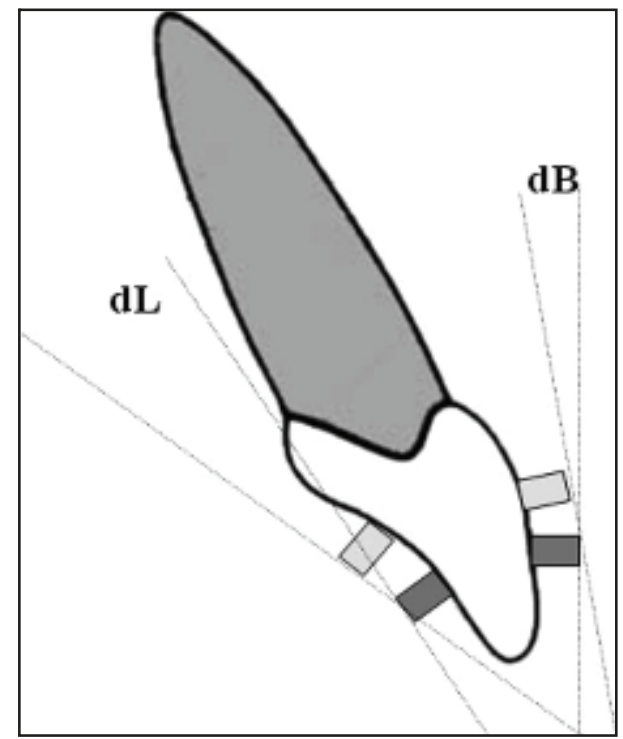

Figure 8: Changing the bracket position on the lingual side creates unpredictable and extensive change in the torque and vertical tooth height. ${ }^{18}$ 
Fifth, the interbracket span, on the other hand, remains another crucial factor affecting the effectiveness of tooth displacement. It has been established that a sufficient interbracket span enables the flexible archwire to activate its springiness caused by deflection. ${ }^{25}$ Regarding Inman Aligner, it gave rise to the lowest mean of tooth tipping and rotation. This is most probably because the labial bow of this appliance has a point contact with the tooth surface, while the conventional buccal technique and lingual appliance has more contact with the tooth surface through the bracket and the archwires, this will result in less torque control. In addition the labial bow of Inman Aligner will drop down soon after activation of the appliance resulting in less controlled tooth movements.

\section{Conclusion}

All the three techniques are efficient in alignment of teeth. The best method of alignment is the conventional buccal technique followed by the lingual technique. The least favourable one is the Inman Aligner.

\section{Conflicts of interest}

The authors report no conflicts of interest.

\section{References}

1. Jena AK, Duggal R, Mehrotra AK. Physical properties and clinical characteristics of ceramic brackets: A comprehensive review. Trends Biomater Artif Organs 2007; 20(2):1.

2. Winchester $L$. Bond strengths of five different ceramic brackets: an in vitro study. Eur $\mathrm{J}$ Orthod 1991; 13:293-305.

3. Harris A, Joseph V, Rossouw P. Shear peel bond strengths of esthetic orthodontic brackets. Am J Orthod Dentofac Orthop 1992; 102:215-9.

4. Angolkar P, Kapila S, Duncanson JMG, Nanda R. Evaluation of friction between ceramic brackets and orthodontic wires of four alloys. Am J Orthod Dentofac Orthop 1990; 98:499-506.

5. Pratten D, Popli K, Gemmane N, Gunsolley J. Frictional resistance of ceramic and stainless steel orthodontic brackets. Am J Orthod Dentofac Orthop 1990; 98:398-403.

6. Nimitpornusko C, Viwattanatipa N. Introduction to lingual orthodontics. KDJ 2000; 3(2):2.

7. Proffit WR, Fields HW, Ackerman JL. Mechanical principles in orthodontic force control. In: Proffit
WR (ed.). Contemporary orthodontics. 2000: $326-62$

8. Qureshi A. The Inman Aligner for Anterior Tooth Alignment. Dent Update 2008; 35:377-84.

9. Huffman DJ, Way DC. A clinical evaluation of tooth movement along arch wires of two different sizes. Am J Orthod Dentofacial Orthop 1983; 83 (6):453-9.

10. Rhee JN, Chun YS and Row J. A comparison between friction and frictionless mechanics with a new typodont simulation system. Am J Orthod Dentofacial Orthop 2001; 119(3):293-9.

11. Alshahery WG. Upper canine retraction by sliding technique using stainless steel and nickel titanium coil spring. M.Sc. thesis, College of Dentistry, university of Mosul; 2010; Pp: 64, 66-71.

12. Kircelli ND. Incorporating the forsus fatigue resistant device with the Incognito appliance system. Orthotown 2013; 17(2):38-41.

13. Yamada K, Kuroda Sh, Deghuchi T, Yamamoto TT, Yashiro T. Distal movement of maxillary molars using miniscrew anchorage in the buccal interradicular region. The Angle Orthodontist 2009; 79(1):78-84.

14. Geron S, Romano R, Brosh T. Vertical forces in labial and lingual orthodontics applied on maxillary incisors. Angle Orthodontist 2004; 74 (2):6-8.

15. Alexander CM, Alexander RG, Gorman JC. Lingual orthodontics: a status report. Part 5. Lingual mechanotherapy. J Clin Orthod 1983; 17 (2):99-115.

16. Smith JR, Gorman JC, Kurz C. Keys to success in lingual therapy. Part 1. J Clin Orthod 1986; 20:252-61.

17. Nanda $R$, Kuhlberg A. Principles of biomechanics. In: Nanda R, ed. Biomechanics in Clinical Orthodontics. Philadelphia, Penn: WB. 1997; 1-22.

18. Stamm T, Wiechmann D, Heinecken A, Ehmer U. Relation between second and third order problems in lingual orthodontic treatment. $J$ Lingual Orthod 2000;1:5-11.

19. Diamond M. Critical aspects of lingual bracket placement. J Clin Orthod 1983;17:688-91.

20. Diamond M. Improved vision and isolation for direct lingual bonding of the upper arch. J Clin Orthod 1984; 18:814-5.

21. Scholz RP, Swartz ML. Lingual orthodontics: a status report. Part 3 . Indirect bonding. J Clin Orthod 1982; 16:812-20.

22. Liaw YC, Su YY, Lai YL, Lee SY. Stiffness and frictional resistance of a superelastic nickel-titanium orthodontic wire with low-stress hysteresis. Am J Orthod and Dentofac Orthop 2007; 131:578.e12-578.e18

23. Rinchuse DJ, Rinchuse DJ, Kapur-Wadhwa R. Orthodontic appliance design. Am J Orthod Dentofacial Orthop 2007; 131:76-82. 
Alignment of teeth using different aesthetic .......

Zanco J. Med. Sci., Vol. 19, No. (3), 2015

http://dx.doi.org/10.15218/zjms.2015.0043

24. Bednar JR, Gruendeman GW. The influence of bracket design on moment production during axial rotation. Am J Orthod Dentofacial Orthop 1998; 104:254-61.

25. Schudy GF, Schudy FF. Intrabracket space and interbracket distance: critical factors in clinical orthodontics. Am J Orthod Dentofacial Orthop 1989; 96:281-94. 